\title{
A Randomized, Controlled Clinical Trial of Autologous Adipose-derived Stem Cell Transplantation to Promote Mechanical Stretch- induced Skin Regeneration
}

\section{Poh-Ching Tan}

Shanghai 9th Peoples Hospital Affiliated to Shanghai Jiaotong University School of Medicine Department of Plastic Surgery

\section{Pei-Chuan Chao}

Shanghai 9th Peoples Hospital Affiliated to Shanghai Jiaotong University School of Medicine Department of Plastic Surgery

\section{Chen Cheng}

Shanghai 9th Peoples Hospital Affiliated to Shanghai Jiaotong University School of Medicine Department of Plastic Surgery

\section{Chu-Hsin Chen}

Shanghai 9th Peoples Hospital Affiliated to Shanghai Jiaotong University School of Medicine Department of Plastic Surgery

\section{Ru-Lin Huang}

Shanghai 9th Peoples Hospital Affiliated to Shanghai Jiaotong University School of Medicine Department of Plastic Surgery

\section{Shuang-Bai Zhou ( $\nabla$ shuangbaizhou@yahoo.com )}

Department of Plastic \& Reconstructive Surgery, Shanghai Ninth People's Hospital, Shanghai Jiao Tong University, 639 Zhizhaoju Road, Shanghai, P.R. China

\section{Yun Xie}

Shanghai 9th Peoples Hospital Affiliated to Shanghai Jiaotong University School of Medicine Department of Plastic Surgery

\section{Qingfeng Li}

Shanghai 9th Peoples Hospital Affiliated to Shanghai Jiaotong University School of Medicine Department of Plastic Surgery

\section{Research}

Keywords: stem cell, stromal vascular fraction (SVF), mechanical stretch, skin regeneration, skin expansion 
Posted Date: November 3rd, 2020

DOI: https://doi.org/10.21203/rs.3.rs-99755/v1

License: (c) (i) This work is licensed under a Creative Commons Attribution 4.0 International License. Read Full License

Version of Record: A version of this preprint was published at Stem Cell Research \& Therapy on April 15th, 2021. See the published version at https://doi.org/10.1186/s13287-021-02318-5. 


\section{Abstract}

Background: The regeneration response of skin to mechanical stretching in vivo has been explored in reconstructive surgery for repairing large-scale deformities. The ability of skin to regenerate limits the reconstructive outcome. Here, we propose an approach in which autologous adipose-derived stem cells and mechanical stretching are combined to overcome this limitation and promote skin regeneration.

Methods: This randomized, blinded, placebo-controlled clinical trial screened 22 participants undergoing tissue expansion with a presence of exhausted regeneration. Twenty eligible participants received intradermal injections with stromal vascular fraction (SVF) or placebo treatments. Follow-ups were conducted at 4, 8, and 12-weeks to assess efficacy and for 2-years to assess safety. The primary endpoint was the expanded skin thickness at 12 weeks. The secondary endpoints included the skin thickness at 4 and 8 weeks, the expansion index (EI) and the skin texture score at all visits.

Results: The skin thickness of the SVF group was significantly higher than that of the control group at both 8 weeks (mean difference 0.78 [95\% $\mathrm{Cl}-1.43$ to -0.11$] ; \mathrm{p}=0.018$ ) and 12 weeks $(0.65[95 \% \mathrm{Cl}-1.30$ to $-0.01] ; p=0.046)$. In the SVF group, the increment of skin thickness was significant at 4 weeks $(0.49$ [95\% $\mathrm{Cl}-0.80$ to -0.06 ]; $p=0.010)$ to 8 weeks $(0.45$ [95\% $\mathrm{Cl}-0.92$ to 0.02 ]; $p=0.026)$ and maintained after 12 weeks, whereas that in the control group was reduced after 8 weeks $(0.42$ [95\% $\mathrm{Cl}-0.07$ to 0.91$] ; p=$ 0.037). The SVF group showed higher El increments than the control group ( 0.50 [95\% Cl -0.00 to 0.99$] ; p$ $=0.047)$. The skin texture scores in the SVF group were higher than those in the control group at 12 weeks. Histologically, the SVF-treated expanded skin showed more proliferating cells and blood vessels, and the volume of extracellular matrix increased. No severe adverse events occurred.

Conclusions: Transplantation of autologous adipose-derived stem cells can expedite the potency of mechanical stretch-induced skin regeneration and provide clinical reconstruction with plentiful tissue.

Trial registration: This trial was registered with Chinese Clinical Trial, ChiCTR2000039317 (registered 23 Oct 2020 - retrospectively registered, http://www.chictr.org.cn/showproj.aspx?proj=62738).

\section{Background}

Repairing large skin defects remains a major clinical challenge due to a lack of suitable tissue. Soft tissue expansion, which induces in vivo skin regeneration via mechanical stretching, is a reliable method for massive skin deficiency reconstruction(1). Continuous mechanical stretch induced by inflating subcutaneous silicone expanders stimulates cell proliferation and extracellular matrix synthesis(2) and creates vascularized skin tissue with an integrated structure and a well-matched texture(3).

However, in patients with massive skin defects, the amount of skin needed often exceeds the amount that can be provide via skin expansion. The growth capacity of skin can only support expansion to two to three times that of the original area. Overexpansion leads to skin thinning, ischemia, and possibly even necrosis(4-6) and severely limits reconstructive outcomes. Furthermore, the skin expansion routine 
usually takes more than 6 months, with an increased incidence of adverse events $(7,8)$. Therefore, new methods for promoting skin regeneration and accelerating inflation are needed to challenge the limitations of soft-tissue reconstruction.

Over the last decade, the excitement about stem cell research has been unprecedented. Adult stem cells are emerging as a promising therapeutic agent for tissue repair and regeneration(9-11). In our preclinical studies, we reported that stem cells can effectively promote mechanical stretch induced skin regeneration by differentiation and growth factors secretion(12-17). Adipose tissue is a large and easily approached depot of stem/progenitor cells. Stromal vascular fractions (SVF), separated from adipose tissue by collagenase digestion, is a heterogeneous cellular population enriched with stem cells and progenitors(18-21). Recent studies reported that SVF participates in ectoderm and endoderm tissue repair and regeneration, including the modulation of inflammation, cell proliferation and ECM synthesis, with the formation of new blood vessels and granulation(22-25). Our preclinical study showed that intradermal transplantation of SVF promoted skin expansion $(14,15)$. In our study, we proposed that SVF treatment promote skin regeneration under mechanical stretching and optimize skin expansion limitations.

In this single-blinded, randomized, controlled clinical trial, we aimed to evaluate the safety and efficacy of intradermal transplantation of autologous SVF in promoting mechanical stretch-induced skin regeneration.

\section{Method Study Design}

The study was a parallel, single-blinded, randomized, controlled clinical trial. Based on data from previous research, with 8 patients per study arm, the statistical power was $>90 \%$ (two-sided, alpha $=0.05$ ). Assuming an attrition rate of $20 \%$ over the course of the study, we enrolled 20 patients in total (10 per group.)

\subsection{Patients}

Patient recruitment began in March, 2014. Eligible participants met the following inclusion criteria: 18 to 65 years old without a gender preference; an indication of deterioration in expanded skin texture, such as thin/papery skin, angiotelectasia, and dermis striatum that showed no improvement after suspending expansion for 2 weeks; and a requirement for further expansion to achieve reconstruction. All patients were assessed by two independent plastic surgeons for eligibility.

The exclusion criteria were as follows: (1) a history of severe illnesses, including any cancers, hepatitis, coronary artery disease, arteriosclerosis, diabetes, and obesity (BMI > 30); (2) presence of infection in the expansion area; (3) presence of expanded skin in a haired area; and (4) current smoking or smoking cessation for less than 6 months before enrollment.

\subsection{Randomization and Masking}


Each eligible patient provided written informed consent and was randomly assigned to the SVF or control group in a 1:1 ratio using a computer-generated randomization schedule. The recruiting team randomized the participants, and the conduct team managed the treatments. The evaluating investigator and data collectors were masked to the group allocation. No substantial changes to the study methodology were made after commencement of the trial.

\subsection{Intervention and Tissue Expansion Protocol}

The SVF was isolated as in our preclinical study $(14,15)$. Briefly, liposuction was performed under local anesthesia with a wet-swelling solution containing lidocaine and adrenaline, and subcutaneous fat was harvested from the abdomen or posterior inner thigh region depending on the amount of fat that was preserved. The fat was aspirated using an 18-gauge liposuction needle connected to a 20-mL syringe with low pressure. The fat was centrifuged at $600 \mathrm{rpm}$ for 2 minutes to remove oil droplets and the swelling solution. Each 20-mL fat sample was incubated with $600 \mathrm{U}$ of collagenase (Shanghai Qiaoyuan Biological Pharmaceutical Co, LTD, Shanghai, China) at $37^{\circ} \mathrm{C}$ for 1 hour. The sample was then filtered and centrifuged at $1500 \mathrm{rpm}$ for 5 minutes to obtain the pelleted SVF. The SVF pellet was suspended with sterile saline twice, and the cell concentration was adjusted to $1 * 10^{6} \mathrm{cells} / \mathrm{ml}$ in 2 -mL syringes. The skin was pinched to facilitate the intradermal injection of the SVF suspension via a 30-G needle, with approximately $0.1 \mathrm{ml}$ injected at a $1-\mathrm{cm}$ interval $\left(1 \times 10^{5} \mathrm{cells} / \mathrm{cm}^{2}\right)$ (Figure 2a,b). The control group was injected with an equal volume of sterile saline in the same manner.

The expander was inflated every 3 days, and a pressure meter was used to monitor the inflation pressure; inflation was discontinued when the inflation pressure reached $100 \mathrm{mmHg}$. This procedure was repeated until sufficient tissue was obtained or the maximal regenerative assessment was achieved to perform skin flap transfer surgery.

\subsection{Assessment and Endpoint}

Outcomes were assessed at baseline (immediately before treatment) and at 4 weeks, 8 weeks, and 12 weeks after the first treatment. The primary endpoint was the change in skin thickness at 12 weeks posttreatment. The secondary endpoints were the changes in the skin thickness including the epidermal and dermal thicknesses, the expansion index (EI), and the subjective scores of skin texture at 4 weeks and 8 weeks during the follow-up period. Safety assessments were administered at 2 years.

\subsubsection{Expanded Skin Thickness}

Skin thickness was measured at eight evenly spaced measurement points along the longitudinal axis using a duplex ultrasonic scanner(26). In ultrasound images, skin appears as a well-defined, linear echogenic band between the air-epidermal band and the low echo-level hypodermal band. The epidermal thickness was defined as the distance between the most superficial, clearly visual high echo-level band, and the dermal thickness was defined as the distance between the moderate-echogenic band underneath the epidermal band. 


\subsubsection{Expansion Index (EI)}

The inflated volumes at each visit were recorded and summated. Due to the maximum capacity of the patient's expanders, we used the El to measure the efficiency of the inflation process; the El was described as the ratio of the total inflated volume $(\mathrm{ml})$ to the designed volume expander $(\mathrm{ml})$.

\subsubsection{Subjective Scores of Expanded Skin Texture}

The investigator-assessed subjective scores of expanded skin texture were as follows: 3 indicated that the skin texture was significantly improved and that the optimal desired result was obtained; 2 indicated that the skin texture was significantly improved, but further treatments were required; 1 indicated that the skin texture was slightly improved; 0 indicated that the skin texture was the same as that before treatment; and -1 indicated that the skin texture was worse than that before treatment. The same blinded investigator assessed all scores.

\subsubsection{Histological Examination}

Expanded skin specimens were harvested during flap transfer surgery. Specimens were fixed with formalin, embedded in paraffin, and sectioned at a thickness of 4- $\mu \mathrm{m}$. Hematoxylin and eosin (HE) staining and Masson staining were performed to assess the histologic differences. Collagen synthesis was evaluated using the collagen volume fraction (CVF). Immunohistochemistry with proliferating cell nuclear antigen (PCNA) was used to evaluate the role of SVF in promoting skin cell proliferation, and the vascular endothelial cells were identified as the surrounding vessels using an anti-CD31 antibody to evaluate angiogenesis in the skin. The number of positive cells was calculated using a laser confocal microscope (Leica, Wetzlar, Germany) in 5 high-power random fields (HPFS, 400X).

\subsubsection{Safety}

During the 2-year follow-up period, the following factors were evaluated to identify adverse reactions: redness, swelling, pain, infectious symptoms and symptoms related to the presence of a subcutaneous protuberance, mass, induration, or hyperplasia.

\subsection{Study Oversight}

This study was granted ethical approval from the Institutional Ethics Committee at the Shanghai Ninth People's Hospital in accordance with the principles of the Declaration of Helsinki. All treatments and follow-up visits were performed at the Department of Plastic and Reconstructive Surgery of Shanghai Ninth People's Hospital, Shanghai Jiaotong University School of Medicine from March 2014 to December 2018. This trial was registered with Chinese Clinical Trial, ChiCTR2000039317 (registered 23 Oct 2020 retrospectively registered, http://www.chictr.org.cn/showproj.aspx?proj=62738).

\subsection{Statistical Analysis}


GraphPad Prism 7 (GraphPad Software Inc., San Diego, CA, USA) was used for the statistical analysis. Continuous data were expressed as the mean \pm standard deviation (SD) with a $95 \%$ confidence interval $(\mathrm{Cl})$, and categorical data were represented as counts. The between-group differences at each visit were performed using two-way repeated-measures ANOVA followed by Sidak's multiple comparison tests, and the within-group differences of each follow-up visit compared to baseline were analyzed using the paired t-test. The El increment between groups was assessed using the unpaired t-test. A value of $P<$ 0.05 indicated that the difference was statistically significant $\left({ }^{*} p<0.05,{ }^{\star *} p<0.01\right)$.

\section{Results}

\subsection{Participant Characteristics}

In total, 22 patients were screened from March 2014 to December 2016. Two patients were excluded from the study, 10 patients were randomly assigned to the treatment group, and 10 were randomly assigned to the placebo group (Figure 1). All patients completed the follow-up visits; none were lost to follow-up. In total, 11 male and 9 female subjects were included, with average ages of $24.5 \pm 4.4$ years in the control group and $26.8 \pm 5.9$ years in the SVF group. The average duration of the expander implantation was 13.5 \pm 5.4 months in the control group and $12.7 \pm 5.7$ months in the SVF group, and the El index was $2.08 \pm 0.68$ in the control group and $2.39 \pm 0.59$ in the SVF group. Baseline demographic and disease characteristics showed no difference between the groups, as shown in Table 1.

\subsection{Primary Outcome}

At baseline, patients from both groups had similar skin thicknesses (control group $2.03(0.48) \mathrm{mm}$ vs. SVF group $1.93(0.38) \mathrm{mm}, \mathrm{p}=0.957)$. At 12 weeks, the SVF group had significantly thicker skin at 12 weeks posttreatment (mean difference 0.65 [95\% Cl-1.30 to -0.01]; $p=0.046$ ) (Table 2). The skin thickness in the SVF group significantly increased at 4 weeks to $2.36(0.65) \mathrm{mm}$ (mean difference 0.49 [95\% Cl -0.80 to -0.06$] ; p=0.010)$, and the increment maintained at 8 weeks $(2.38(0.59) \mathrm{mm}$ (mean difference 0.45 [ $95 \% \mathrm{Cl}-0.92$ to 0.02$] ; p=0.026)$ ). In the control group, the skin thickness deteriorated after 4 weeks. At 12 weeks, the skin thickness of the SVF group was still higher than that at baseline (2.14 $(0.54) \mathrm{mm}$ ), while that of the control group was significantly decreased $(1.48(0.50) \mathrm{mm})$ (Figure 2$)$.

The main contribution to skin thickness changes was from the dermis. The epidermal thickness was similar in the two groups. Compared to the baseline, the dermis in the SVF group was significantly thickened at 4 weeks $(1.86(0.59) \mathrm{mm}$ ) (mean difference 0.37 [95\% $\mathrm{Cl}-0.77$ to 0.03 ]; $\mathrm{p}=0.029$ ) and 8 weeks $(1.82(0.55) \mathrm{mm}$ ) (mean difference 0.33 [95\% $\mathrm{Cl}-0.73$ to 0.08]; $\mathrm{p}=0.048$ ). Meanwhile, the thickness of the dermis in the control group decreased continuously (Figure 2). These findings indicate that SVF treatment could promote skin regeneration by increasing the dermal thickness to prevent the expanded skin from becoming papery; in contrast, the control group exhibited thinning of the skin after inflation. 


\subsection{Secondary Outcome}

\subsubsection{Expansion Index (EI)}

The El increment from baseline to 12 weeks was significantly increased in the SVF group $(0.940(0.698)$ versus the control group $(0.440(0.255)$ ) (mean difference 0.50 [95\% $\mathrm{Cl}-0.00$ to 0.99$] ; p=0.047$ ) (Figure $3)$. The results indicate that the SVF group had higher inflation volumes and larger skin areas at the end of follow-up.

\subsubsection{Skin Texture Evaluation}

The blinded investigator subjectively evaluated the skin texture scores at 12 weeks. The SVF group showed significant improvement in skin texture compared to the control group, as the number of patients scored as "significantly improved" and "improved" was higher in the SVF group (3-grade: 4 [40\%] and 2grade: 2 [20\%] vs. 3-grade: 0 [0\%] and 2-grade: 1 [10\%]) (Figure 4a). The improved skin textures in the SVF group at their 12-week assessment are highlighted in Figure 4. Fewer patients in the treatment group complained of thin, papery/transparent skin compared to the control group during the follow-up.

\subsubsection{Histological Examination}

The HE staining results show that the epidermal layer in the SVF group was thicker than that in the control group, with the presence of an increasing rete subpapillary in the papillary layer (Figure $5 a, b)$. The papillary dermis was thickened, and the collagen fibers were increased with an organized distribution in the SVF group, while the collagen fibers in the control group were loosely organized (Figure $5 c, d$ ). Masson's trichrome staining showed that the CVF in the SVF group was 54.1(19.9) \%/HPF, which is significantly higher than that in the control group (71.1(14.7) \%/HPF, $p=0.049$ ) (Figure 5i).

Compared with the control group, the number of PCNA + proliferating cells was significantly higher in the SVF group (28.1(19.3)/HPF versus 55.1(33.9)/HPF in the control group, $p=0.042$ ) (Figure 5j). The proliferating cells in the SVF group presented not only in the basal layer of the epidermis but also in the papillary layer of the dermis (Figure $5 e, f)$. Higher vascular density throughout the dermis was observed in the SVF group (Figure 5g,h) (17.2(6.5)/HPF versus 26.6(12.2)/HPF in the control group, $p=0.045$ ) (Figure $5 k)$, indicating improved vascularization after SVF injection.

\subsubsection{Safety}

After the injection, 12 participants (60\%) had temporary ecchymosis in the injection area; however, the symptoms disappeared within a week without sequelae. None of the participants had infectious or severe adverse events during the 24-week follow-up period. During the 2-year follow-up, no subcutaneous protrusion, mass, induration, or hyperplasia were found.

\section{Discussion}


Stimulating skin with mechanical stretching induces a cellular response and results in extracellular matrix synthesis, vascularization, and the proliferation of fibroblasts and epidermis cells(27-29). Skin expansion is the application of mechanical stretch-induced skin regeneration in reconstructive surgery for gaining regenerated tissue(2). However, the expansion of skin is limited by the intrinsic capacity of skin to regenerate $(4,6)$. Numerous studies have explored methods to promote skin regeneration, including the use of prostaglandin E2, papaverine, cytochalasin, and dimethyl sulfoxide (DMSO) for expediting skin expansion, but no study has established sufficient clinical efficacy(30-32). The mechanism of regenerative exhaustion in skin is still debated and recent findings suggest that stem cell deregulation or insufficiency is involved $(33,34)$. Therefore, we need to explore new methods for promoting tissue expansion by enhancing skin regeneration.

In our previous research(13-16, 35), we found that combining stem-cell therapy with mechanical stretchinduced skin regeneration could overcome the limitation of stretch-induced regeneration. In animal studies, we proved that stem cells from both bone marrow and adipose tissue could improve skin regeneration. The efficacy of bone marrow stem cells in rescuing thinning expanded skin was proven by our previous randomized clinical trial(26). However, harvesting mononuclear cells (MNC) is a painful procedure that produces only a limited number of cells. In contrast, large amounts of SVF can be obtained through a minimally invasive liposuction procedure, suggesting that SVF cells are more appropriate as the source of cell-based therapy in clinical applications. Hence, in this research, we discussed the clinical efficacy and safety of SVF in stimulating mechanical stretch-induced tissue regeneration.

The results from this study indicate that intradermal transplantation of SVF aids the sustainable regeneration of expanded skin that shows signs of regenerative exhaustion. The SVF group showed statistically significant improvement versus both the control group and the baseline for primary and secondary outcomes. After SVF treatment, the thickness of the expanded skin significantly increased at 4 and 8 weeks after treatment and maintained at 12 weeks. In the control group, the skin thickness showed a continuous decrease during the follow up. Approximately $60 \%$ of the cases in the SVF group were evaluated as having an improved texture, while only $10 \%$ of the control group had a better skin texture than the baseline. Our results show that intradermal injection of SVF reversed the thinning and deterioration of expanded skin and helped continuing skin regeneration.

SVF participates in tissue regeneration via multiple mechanisms $(17,18)$ involved in anti-apoptotic, immunomodulatory, anti-inflammatory, and angiogenesis actions(36). Endothelial progenitor cells (EPCs) and adipose-derived stem cells (ADSCs) may contribute to these actions. EPCs served as the endothelial progenitors, endothelial cells, and pericytes, which contributed to neovascularization and vessel remodeling $(37,38)$. ADSCs can secrete the growth factor and chemotaxis, such as the vascular endothelial growth factor (VEGF), fibroblast growth factor -2(FGF-2), hepatocyte growth factor (HGF), and interleukin 6 (IL-6), which can involve regulating immune, anti-inflammatory, and angiogenesis responses $(19,20)$. The coculture of ADSCs and ECs increased the production of ECM, which stabilized the newly formed vessels(39). Studies have shown that SVF treatment enhances the density of 
capillaries, expedites the healing process in the wounds of murine, and promotes vascularization of ischemic limbs in the murine model(40-42). In our study, the histological results of our trial show that more blood vessels were observed in tissue from the SVF group. Additionally, the numbers of proliferating basal cells and fibroblasts increased as the volume of extracellular matrix (ECM) increased.

SVF treatment delivers a better therapeutic effect without increasing adverse events. No subcutaneous protrusion, mass, induration, or hyperplasia were found in the SVF group. Although the ecchymosis spontaneously resolved, it decreased the satisfaction and compliance of the participants.

As in our previous study(26), MNC treatment increased the skin thickness after 4 weeks, but the increment consistently declined after eight weeks following inflation. Compared to SVF treatment, the therapeutic effect of the cellular component of SVF is more lasting in mechanical stretch-induced skin regeneration.

There are still some limitations in our study. Further randomized controlled trials with larger sample sizes and longer follow-up durations are warranted. The potential immunogenicity and tumorigenicity of the SVF treatment must be investigated before clinical use. We need to further explore the cellular mechanism of SVF transplantation in promoting skin regeneration under mechanical stretching. Although the histological results showed more proliferating cells and angiogenesis in the SVF treatment group, the transplanted stem cells could not be tracked in vivo.

\section{Conclusion}

This clinical trial demonstrated that the application of SVF is reliable and efficient in overcoming the limitations of mechanical stretch-induced skin regeneration. The SVF group showed a better outcome than the control group in expediting the potency of skin expansion, increasing the dermal thickness, and ensuring a sufficient inflation volume. We believe that the synergistic combination of stem cells and mechanical stretching stimulation will promote the further development of tissue regeneration in vivo and help to achieve more satisfactory reconstruction outcomes.

\section{List Of Abbreviations}

Cl囚confidence interval

CVF: collagen volume fraction

DMSO: dimethyl sulfoxide

ECM: extracellular matrix

El: expansion index

HE: Hematoxylin and eosin

MNC: mononuclear cells 
PCNA: proliferating cell nuclear antigen

SD: standard deviation

SVF: stromal vascular fraction

\section{Declarations}

\section{Ethics approval and consent to participate:}

This study was approved by the Ethics Committee of Shanghai Ninth People's Hospital and complied with the principles of the Declaration of Helsinki. All participants provided written informed consent to participate this clinical trial

\section{Consent for publication:}

Informed consent was obtained from all individual participants included in the study.

\section{Availability of data and material:}

The datasets used and/or analyzed during the current study are available from the corresponding author on reasonable request.

\section{Competing interests:}

The authors declare that there are no conflicts of interest regarding the publication of this paper.

\section{Funding:}

This clinical trial was supported by the National Natural Science Foundation of China (No.81971848; 81620108019; 81701917), the Clinical Research Program of Shanghai Jiao Tong University School of Medicine (DLY201509), the Shanghai Pujiang Program (2019PJD024), the Natural Science Foundation of Shanghai (17ZR1416500) and the Two-hundred Talent (20191916).

\section{Authors' contributions:}

SBZ, YX and QFL initiated and designed the study and protocol. CC and PCC recruited all patients. RLH, SBZ and YX participated as treating investigator and gave all injections. $\mathrm{CHC}$ and $\mathrm{PCC}$ participated as evaluating investigator. PCT contributed in data collection, data analyzation and data interpreted, and wrote the first draft of the manuscript. QFL and SBZ critiqued and modified the manuscript. All authors reviewed and approved the work.

\section{Acknowledgements}

Not applicable 


\section{References}

1. Neumann CG. The expansion of an area of skin by progressive distention of a subcutaneous balloon; use of the method for securing skin for subtotal reconstruction of the ear. Plast Reconstr Surg (1946). 1957;19(2):124-30.

2. Aragona M, Sifrim A, Malfait M, Song Y, Van Herck J, Dekoninck S, et al. Mechanisms of stretchmediated skin expansion at single-cell resolution. Nature. 2020;584(7820):268-73.

3. Marcus J, Horan DB, Robinson JK. Tissue expansion: past, present, and future. J Am Acad Dermatol. 1990;23(5 Pt 1):813-25.

4. Pamplona DC, Velloso RQ, Radwanski HN. On skin expansion. J Mech Behav Biomed Mater. 2014;29:655-62.

5. Zan T, Gao Y, Li H, Gu B, Xie F, Li Q. Pre-expanded, Prefabricated Monoblock Perforator Flap for Total Facial Resurfacing. Clin Plast Surg. 2017;44(1):163-70.

6. Jahed Z, Shams H, Mehrbod M, Mofrad MR. Mechanotransduction pathways linking the extracellular matrix to the nucleus. Int Rev Cell Mol Biol. 2014;310:171-220.

7. Wang J, Huang X, Liu K, Gu B, Li Q. Complications in tissue expansion: an updated retrospective analysis of risk factors. Handchir Mikrochir Plast Chir. 2014;46(2):74-9.

8. Huang X, Qu X, Li Q. Risk factors for complications of tissue expansion: a 20-year systematic review and meta-analysis. Plast Reconstr Surg. 2011;128(3):787-97.

9. Dresser R. Stem cell research as innovation: expanding the ethical and policy conversation. J Law Med Ethics. 2010;38(2):332-41.

10. Wahid FSA, Ismail NA, Wan Jamaludin WF, Muhamad NA, Mohamad Idris MA, Lai NM. Efficacy and Safety of Autologous Cell-based Therapy in Patients with No-option Critical Limb Ischaemia: A MetaAnalysis. Curr Stem Cell Res Ther. 2018;13(4):265-83.

11. Kumar A, Prasad M, Jali VP, Pandit AK, Misra S, Kumar P, et al. Bone marrow mononuclear cell therapy in ischaemic stroke: a systematic review. Acta Neurol Scand. 2017;135(5):496-506.

12. Li H, Zan T, Li Y, Weng R, Yang M, Du Z, et al. Transplantation of adipose-derived stem cells promotes formation of prefabricated flap in a rat model. Tohoku J Exp Med. 2010;222(2):131-40.

13. Yang M, Li Q, Sheng L, Li H, Weng R, Zan T. Bone marrow-derived mesenchymal stem cells transplantation accelerates tissue expansion by promoting skin regeneration during expansion. Ann Surg. 2011;253(1):202-9.

14. Sheng L, Yang M, Du Z, Yang Y, Li Q. Transplantation of stromal vascular fraction as an alternative for accelerating tissue expansion. J Plast Reconstr Aesthet Surg. 2013;66(4):551-7.

15. Sheng L, Yang M, Liang Y, Li Q. Adipose tissue-derived stem cells (ADSCs) transplantation promotes regeneration of expanded skin using a tissue expansion model. Wound Repair Regen. 2013;21(5):746-54.

16. Zhou SB, Chiang CA, Liu K, Li QF. Intravenous transplantation of bone marrow mesenchymal stem cells could effectively promote vascularization and skin regeneration in mechanically stretched skin. 
Br J Dermatol. 2015;172(5):1278-85.

17. Liang X, Huang X, Zhou Y, Jin R, Li Q. Mechanical Stretching Promotes Skin Tissue Regeneration via Enhancing Mesenchymal Stem Cell Homing and Transdifferentiation. Stem Cells Transl Med. 2016;5(7):960-9.

18. Sun Y, Chen S, Zhang X, Pei M. Significance of Cellular Cross-Talk in Stromal Vascular Fraction of Adipose Tissue in Neovascularization. Arterioscler Thromb Vasc Biol. 2019;39(6):1034-44.

19. Merfeld-Clauss S, Gollahalli N, March KL, Traktuev DO. Adipose tissue progenitor cells directly interact with endothelial cells to induce vascular network formation. Tissue Eng Part A. 2010;16(9):2953-66.

20. Rehman J, Traktuev D, Li J, Merfeld-Clauss S, Temm-Grove CJ, Bovenkerk JE, et al. Secretion of angiogenic and antiapoptotic factors by human adipose stromal cells. Circulation. 2004;109(10):1292-8.

21. Koh YJ, Koh BI, Kim H, Joo HJ, Jin HK, Jeon J, et al. Stromal vascular fraction from adipose tissue forms profound vascular network through the dynamic reassembly of blood endothelial cells. Arterioscler Thromb Vasc Biol. 2011;31(5):1141-50.

22. Ma T, Sun J, Zhao Z, Lei W, Chen Y, Wang X, et al. A brief review: adipose-derived stem cells and their therapeutic potential in cardiovascular diseases. Stem Cell Res Ther. 2017;8(1):124.

23. Ntege EH, Sunami H, Shimizu Y. Advances in regenerative therapy: A review of the literature and future directions. Regen Ther. 2020;14:136-53.

24. Mazini L, Rochette L, Admou B, Amal S, Malka G. Hopes and Limits of Adipose-Derived Stem Cells (ADSCs) and Mesenchymal Stem Cells (MSCs) in Wound Healing. Int J Mol Sci. 2020;21(4).

25. Goodarzi P, Alavi-Moghadam S, Sarvari M, Tayanloo Beik A, Falahzadeh K, Aghayan H, et al. Adipose Tissue-Derived Stromal Cells for Wound Healing. Adv Exp Med Biol. 2018;1119:133-49.

26. Zhou SB, Zhang GY, Xie Y, Zan T, Gan YK, Yao CA, et al. Autologous Stem Cell Transplantation Promotes Mechanical Stretch Induced Skin Regeneration: A Randomized Phase I/II Clinical Trial. EBioMedicine. 2016;13:356-64.

27. Johnson TM, Lowe L, Brown MD, Sullivan MJ, Nelson BR. Histology and physiology of tissue expansion. J Dermatol Surg Oncol. 1993;19(12):1074-8.

28. Baker SR. Fundamentals of expanded tissue. Head Neck. 1991;13(4):327-33.

29. Beauchene JG, Chambers MM, Peterson AE, Scott PG. Biochemical, biomechanical, and physical changes in the skin in an experimental animal model of therapeutic tissue expansion. J Surg Res. 1989;47(6):507-14.

30. Lee P, Squier CA, Bardach J. Enhancement of tissue expansion by anticontractile agents. Plast Reconstr Surg. 1985;76(4):604-10.

31. Raposio E, Santi PL. Topical application of DMSO as an adjunct to tissue expansion for breast reconstruction. Br J Plast Surg. 1999;52(3):194-7. 
32. Tang $Y$, Luan J, Zhang X. Accelerating tissue expansion by application of topical papaverine cream. Plast Reconstr Surg. 2004;114(5):1166-9.

33. Clevers $\mathrm{H}$, Loh KM, Nusse R. Stem cell signaling. An integral program for tissue renewal and regeneration: Wnt signaling and stem cell control. Science. 2014;346(6205):1248012.

34. Pellettieri J, Sanchez Alvarado A. Cell turnover and adult tissue homeostasis: from humans to planarians. Annu Rev Genet. 2007;41:83-105.

35. Zhou SB, Wang J, Chiang CA, Sheng LL, Li QF. Mechanical stretch upregulates SDF-1alpha in skin tissue and induces migration of circulating bone marrow-derived stem cells into the expanded skin. Stem Cells. 2013;31(12):2703-13.

36. Andia I, Maffulli N, Burgos-Alonso N. Stromal vascular fraction technologies and clinical applications. Expert Opin Biol Ther. 2019;19(12):1289-305.

37. Bora P, Majumdar AS. Adipose tissue-derived stromal vascular fraction in regenerative medicine: a brief review on biology and translation. Stem Cell Res Ther. 2017;8(1):145.

38. Panina YA, Yakimov AS, Komleva YK, Morgun AV, Lopatina OL, Malinovskaya NA, et al. Plasticity of Adipose Tissue-Derived Stem Cells and Regulation of Angiogenesis. Front Physiol. 2018;9:1656.

39. Rohringer S, Hofbauer $P$, Schneider KH, Husa AM, Feichtinger G, Peterbauer-Scherb A, et al. Mechanisms of vasculogenesis in 3D fibrin matrices mediated by the interaction of adipose-derived stem cells and endothelial cells. Angiogenesis. 2014;17(4):921-33.

40. Zhao X, Guo J, Zhang F, Zhang J, Liu D, Hu W, et al. Therapeutic application of adipose-derived stromal vascular fraction in diabetic foot. Stem Cell Res Ther. 2020;11(1):394.

41. Chae DS, Han S, Son M, Kim SW. Stromal vascular fraction shows robust wound healing through high chemotactic and epithelialization property. Cytotherapy. 2017;19(4):543-54.

42. Jin E, Chae DS, Son M, Kim SW. Angiogenic characteristics of human stromal vascular fraction in ischemic hindlimb. Int J Cardiol. 2017;234:38-47.

\section{Tables}

Table 1 Patient demographic and baseline characteristics. 


\begin{tabular}{|c|c|c|c|c|}
\hline & & Control group $\square n=10 \square$ & $\begin{array}{l}\text { SVF group } \\
\square n=10 \square\end{array}$ & $P$ value \\
\hline Age & & & & 0.336 \\
\hline \multirow[t]{2}{*}{ Gender } & Male & 6 & 5 & - \\
\hline & Female & 4 & 5 & - \\
\hline Body Mass Index (BMI) & & & & 0.307 \\
\hline \multirow[t]{4}{*}{ Expander Implantation Site } & Cervical & 7 & 2 & - \\
\hline & Chest & 1 & 4 & - \\
\hline & Dorsal & 0 & 3 & - \\
\hline & Facial & 2 & 1 & - \\
\hline \multirow[t]{2}{*}{ Expander Implantation Time (Month) } & & & & 0.751 \\
\hline & & & & - \\
\hline Expansion Index (EI) & & & & 0.993 \\
\hline \multirow[t]{2}{*}{ Subcutaneous Fat Donor Area } & Abdomen & 4 & 5 & - \\
\hline & Hip & 6 & 5 & - \\
\hline Number of Transplanted Cells $\left(10^{6}\right)$ & & - & & - \\
\hline
\end{tabular}

Table 2 Skin thickness and El parameter at baseline(V1), and after 4 (V2), 8 (V3), 12 (V4) weeks. 


\begin{tabular}{|c|c|c|c|c|c|c|c|c|c|c|c|}
\hline \multirow[t]{2}{*}{ Group } & \multicolumn{5}{|c|}{ Control Group } & \multicolumn{6}{|c|}{ VF Group } \\
\hline & & Mean & $\pm S D$ & \multicolumn{2}{|c|}{$\begin{array}{l}\text { P value } \\
\text { (Visit V.S. V1) }\end{array}$} & Mean & $\pm S D$ & \multicolumn{2}{|c|}{$\begin{array}{l}\text { P value } \\
\text { (Visit V.S. V1) }\end{array}$} & \multicolumn{2}{|c|}{$\begin{array}{l}\text { P value } \\
\text { CON V.S. SVF }\end{array}$} \\
\hline \multirow{4}{*}{$\begin{array}{l}\text { Skin } \\
\text { Thickness } \\
(\mathrm{mm})\end{array}$} & V1 & 2.03 & 0.48 & - & - & 1.91 & 0.42 & - & - & 0.981 & ns \\
\hline & V2 & 1.84 & 0.49 & 0.150 & ns & 2.36 & 0.65 & 0.010 & * & 0.214 & ns \\
\hline & V3 & 1.61 & 0.45 & 0.037 & * & 2.38 & 0.59 & 0.026 & * & 0.018 & * \\
\hline & V4 & 1.48 & 0.50 & 0.008 & ** & 2.14 & 0.54 & 0.144 & ns & 0.046 & * \\
\hline \multirow{4}{*}{$\begin{array}{l}\text { Epidermal } \\
\text { Thickness } \\
(\mathrm{mm})\end{array}$} & V1 & 0.48 & 0.10 & - & - & 0.44 & 0.15 & - & - & 0.900 & ns \\
\hline & V2 & 0.46 & 0.09 & 0.710 & ns & 0.49 & 0.09 & 0.095 & ns & 0.936 & ns \\
\hline & V3 & 0.45 & 0.07 & 0.534 & ns & 0.56 & 0.10 & 0.086 & ns & 0.062 & ns \\
\hline & V4 & 0.44 & 0.10 & 0.315 & ns & 0.51 & 0.10 & 0.251 & ns & 0.421 & ns \\
\hline \multirow{4}{*}{$\begin{array}{l}\text { Dermal } \\
\text { Thickness } \\
(\mathrm{mm})\end{array}$} & V1 & 1.55 & 0.47 & - & - & 1.49 & 0.35 & - & - & 0.997 & ns \\
\hline & V2 & 1.37 & 0.45 & 0.135 & ns & 1.86 & 0.59 & 0.029 & * & 0.199 & ns \\
\hline & V3 & 1.15 & 0.42 & 0.031 & * & 1.82 & 0.55 & 0.048 & * & 0.028 & * \\
\hline & V4 & 1.04 & 0.49 & 0.007 & $* \star$ & 1.64 & 0.46 & 0.189 & ns & 0.045 & * \\
\hline \multirow[t]{2}{*}{ El Index } & V1 & 2.39 & 0.57 & - & - & 2.39 & 0.47 & - & - & 1.000 & ns \\
\hline & V4 & 2.83 & 0.55 & 0.000 & $\star \star$ & 3.06 & 0.79 & 0.002 & $\star *$ & 0.209 & ns \\
\hline $\begin{array}{l}\text { Increment } \\
\text { El }\end{array}$ & $\begin{array}{l}\text { V4- } \\
\text { V1 }\end{array}$ & 0.44 & 0.25 & - & - & 0.94 & 0.70 & - & - & 0.047 & * \\
\hline
\end{tabular}

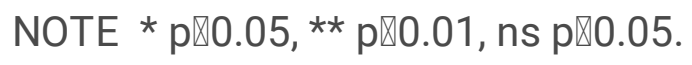




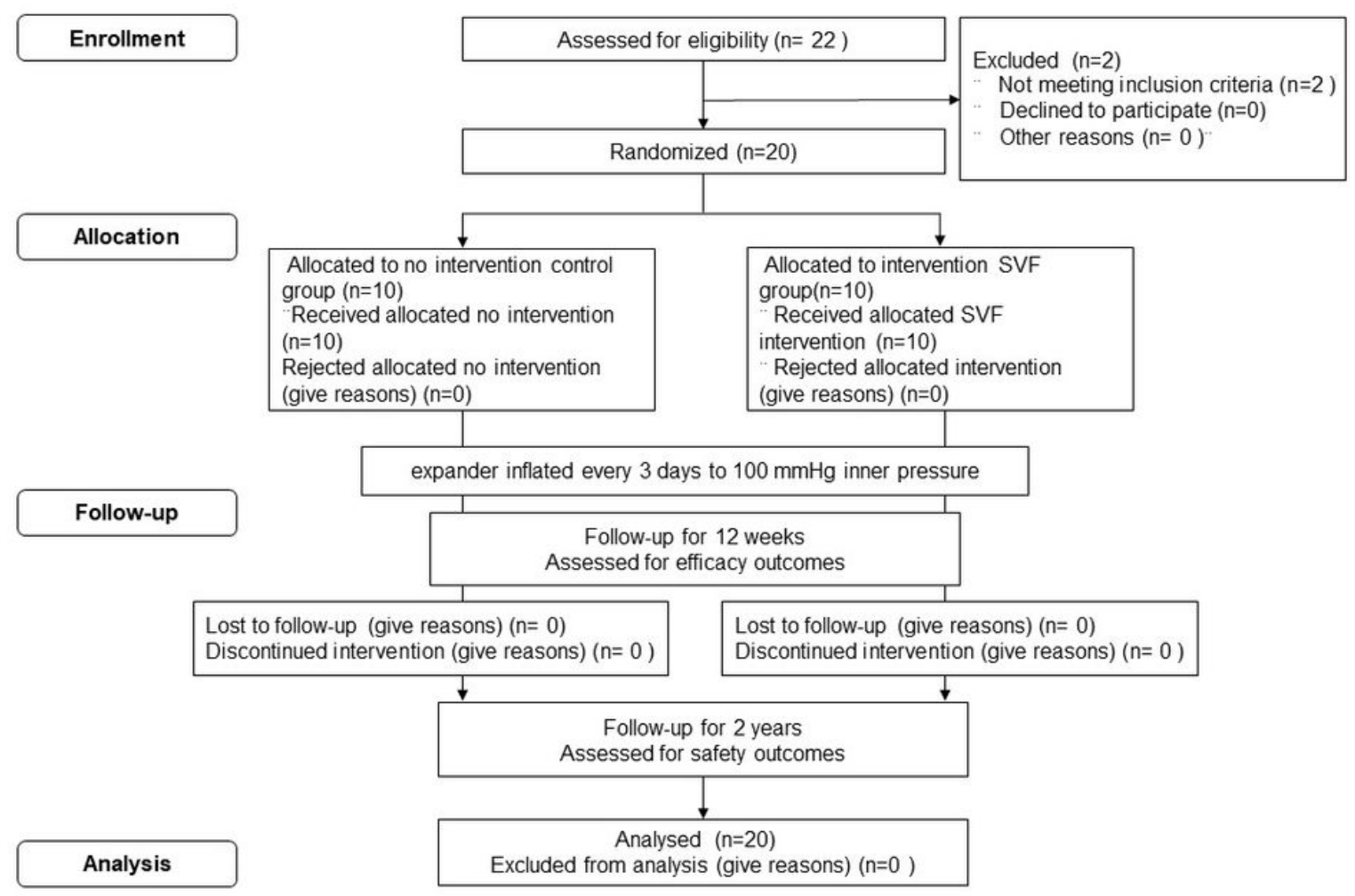

\section{Figure 1}

CONSORT Flow Diagram of participant recruitment.
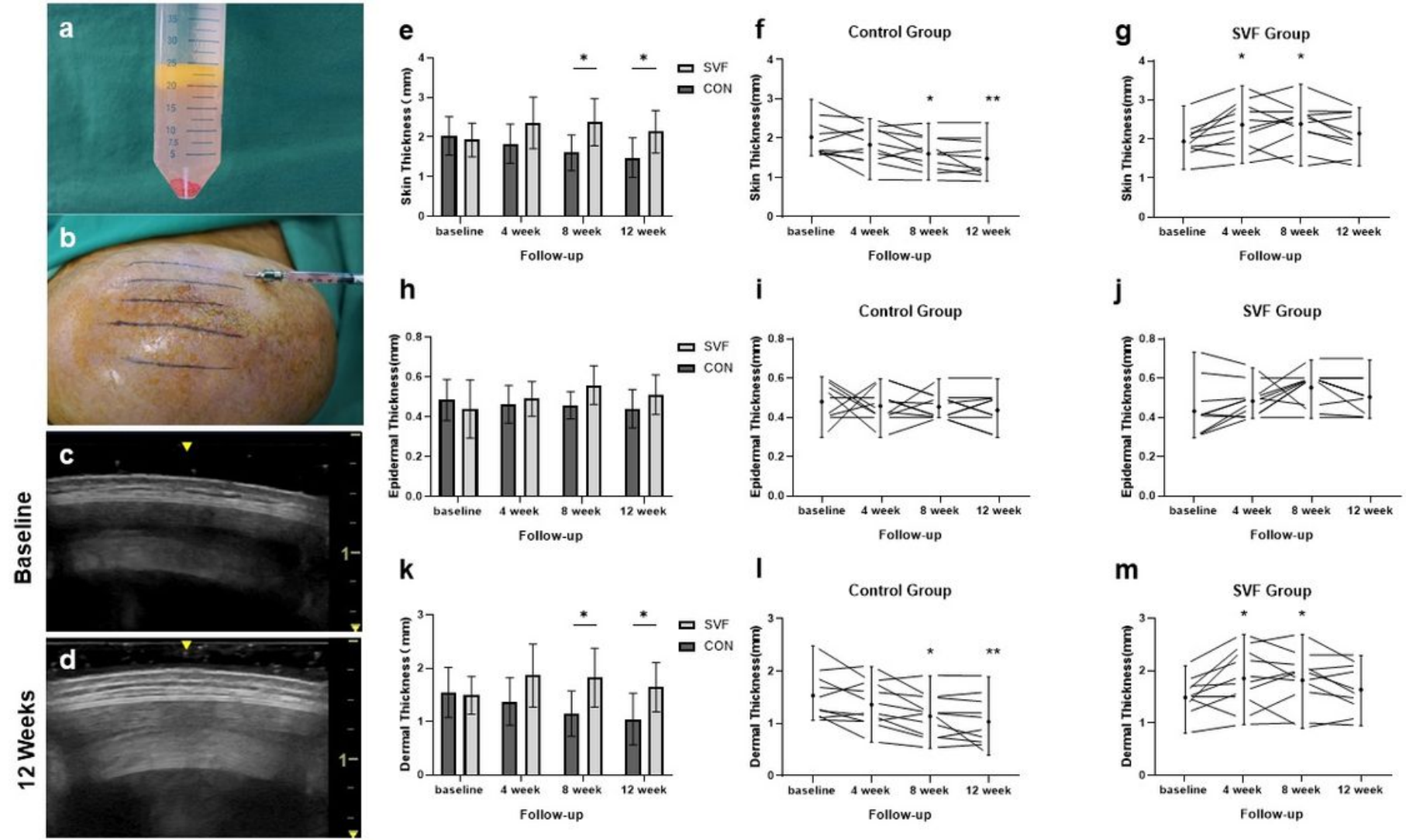

Figure 2 
Changes in skin thickness. Through liposuction and enzyme digestion, the red SVF cell-pellet was harvested (a). SVF was subcutaneously injected onto the expanded skin area (b). An ultrasound was used to assess the skin thickness $(b, c)$ (results from the SVF group at baseline and 12 weeks). The SVF group had a significant increase in the skin thickness compared to the control group at 8 weeks to 12 weeks (e). Compared to the baseline, the SVF group increased significantly at 4 to 8 weeks and was maintained after 12 weeks, whereas the control group decreased after 8 weeks $(f, g)$. A similar downward trend was found for the dermal thickness $(i-k)$, whereas there were no differences in the epidermal thickness between both groups $(I-n) .{ }^{\star} p \otimes 0.05 ; * \star p \otimes 0.01$.

a
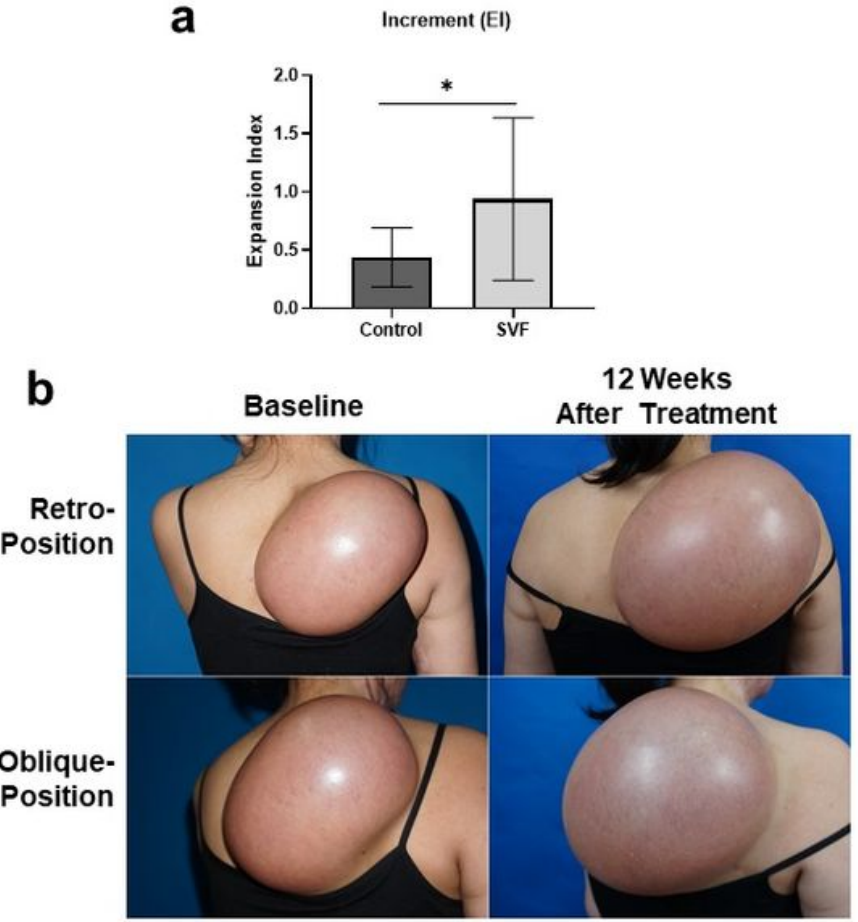

C

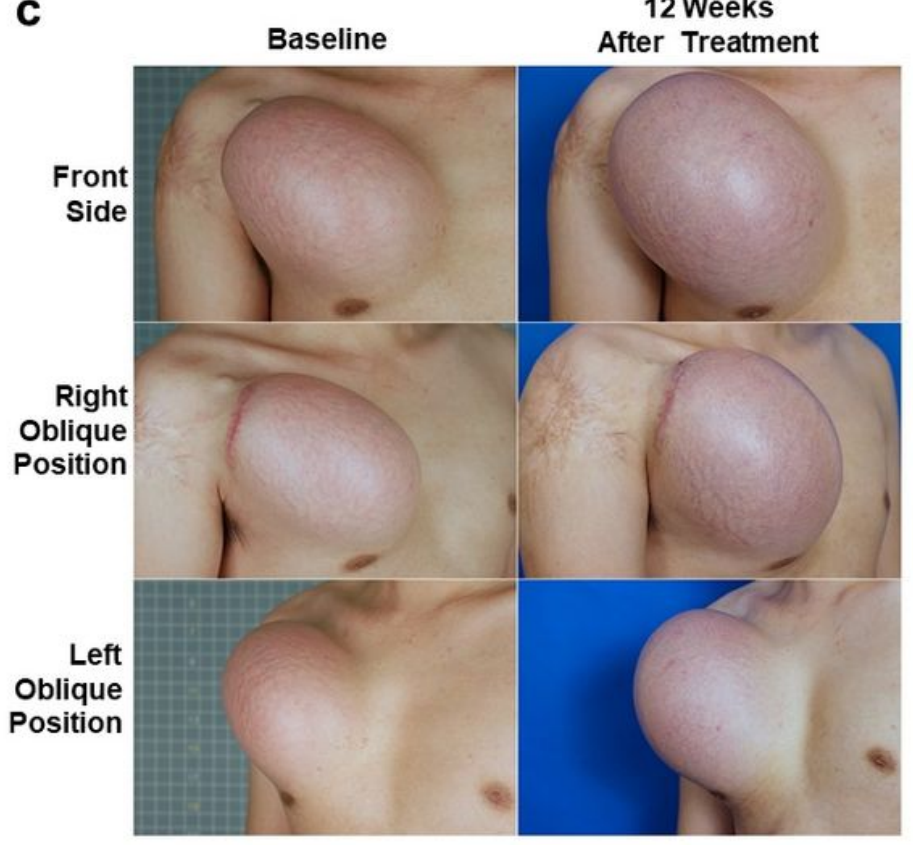

\section{Figure 3}

Changes in the El. The increment of El was significantly increased in the SVF group (a). Patients in the

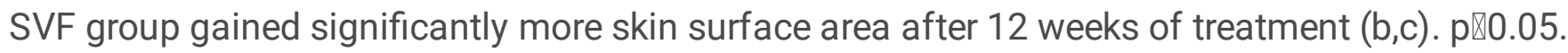


a Subjective Scores
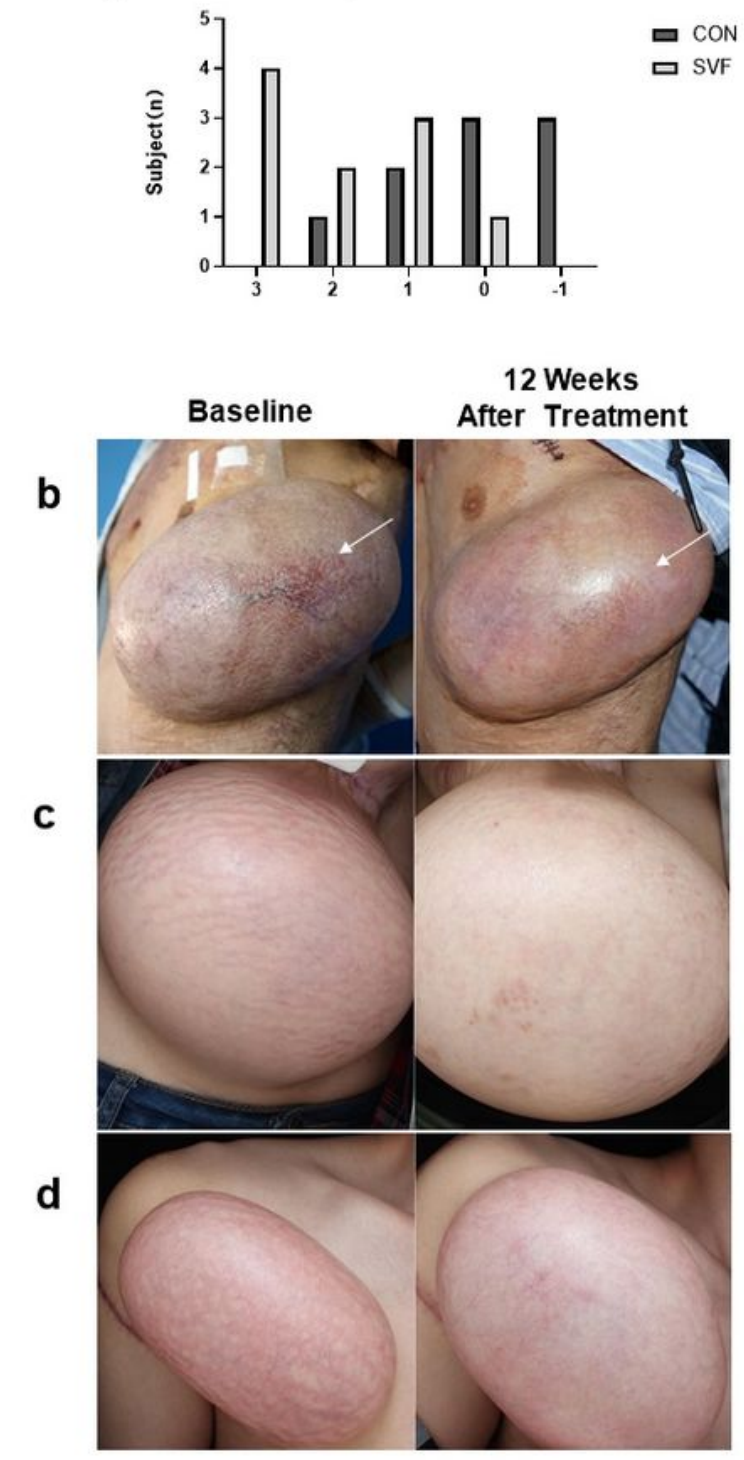

\section{Figure 4}

Evaluation of skin texture. Patients in the SVF group had higher scores when their expanded skin texture was assessed compared to patients in the control group at 12 weeks (a). Patients in the SVF group showed significantly improved skin textures at 12 weeks posttreatment compared to baseline (b-d). (b) Prior to study enrollment, the patient had papery skin accompanied by telangiectasia and the development of an embolism and was facing expansion failure. After the SVF treatment, the texture of his 
skin had improved, and its thickness had increased. The telangiectasia area was diminished. The patient gained further expansion until reaching the need for reconstruction. (c) The patient had stretch marks throughout the expanded skin at baseline. After 12 weeks of treatment, the stretch striae had disappeared and skin growth improved. (d) Before study enrollment, the patient's expanded skin had exhibited papery with stretch striae and could not continue expansion. After 12 weeks of treatment, the skin had increased in thickness and stretch marks diminished.

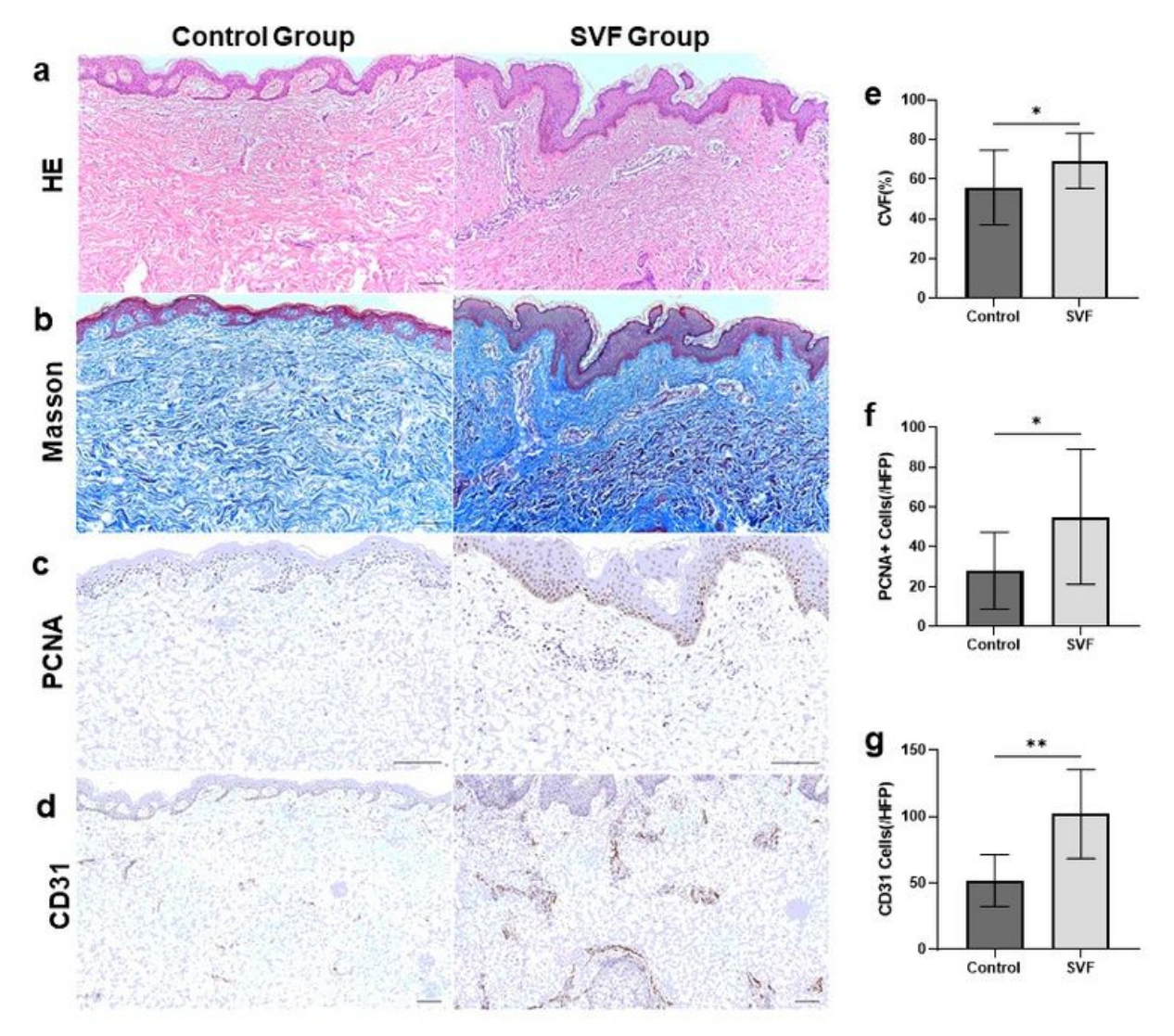

\section{Figure 5}

Histology and immunohistochemical staining of expanded skin. HE staining showed that the epidermal layer in the SVF group was thicker than that in the control group (a), with the presence of an increasing rete subpapillary in the papillary layer. Masson's trichrome staining showed that the ECM volumizing in the SVF group was significantly increased, as the collagen fibers showed an organized distribution, while the collagen fibers in the control group (b) were loosely organized. PCNA + proliferating cells and CD31+ stained vessels were significantly increased in the SVF group compared to the control group $(c, d)$. The results of the statistical analysis showed more proliferating cells (e) and blood vessels (f) in the SVFtreated skin as the volume of ECM increased $(\mathrm{g})$.

\section{Supplementary Files}

This is a list of supplementary files associated with this preprint. Click to download.

- CONSORT2010Checklist.doc 